\title{
An Advocacy for the Use of 3D Stem Cell Culture Systems for the Development of Regenerative Medicine: An Emphasis on Photoreceptor Generation
}

\author{
Yvan Arsenijevic* and Sarah Decembrini
}

Unit of Gene Therapy and Stem Cell Biology, Jules-Gonin Eye Hospital, University of Lausanne, Switzerland

\begin{abstract}
The availability of stem cells is of great promise to study early developmental stages and to generate adequate cells for cell transfer therapies. Although many researchers using stem cells were successful in dissecting intrinsic and extrinsic mechanisms and in generating specific cell phenotypes, few of the stem cells or the differentiated cells show the capacity to repair a tissue. Advances in cell and stem cell cultivation during the last years made tremendous progress in the generation of bona fide differentiated cells able to integrate into a tissue after transplantation, opening new perspectives for developmental biology studies and for regenerative medicine. In this review, we focus on the main works attempting to create in vitro conditions mimicking the natural environment of CNS structures such as the neural tube and its development in different brain region areas including the optic cup. The use of protocols growing cells in 3D organoids is a key strategy to produce cells resembling endogenous ones. An emphasis on the generation of retina tissue and photoreceptor cells is provided to highlight the promising developments in this field. Other examples are presented and discussed, such as the formation of cortical tissue, the epithelial gut or the kidney organoids.
\end{abstract}

The generation of differentiated tissues and well-defined cell phenotypes from embryonic stem (ES) cells or induced pluripotent cells (iPSCs) opens several new strategies in the field of biology and regenerative medicine. A $3 \mathrm{D}$ organ/tissue development in vitro derived from human cells brings a unique tool to study human cell biology and pathophysiology of an organ or a specific cell population. The perspective of tissue repair is discussed as well as the necessity of cell banking to accelerate the progress of this promising field.

Keywords: iPSCs; Retina; Transplantation; Kidney; Gut; Brain regionalization; Cortex; CNS development; Personalized medicine

\section{Introduction}

The possibility to grow in vitro a specific cell population brings a broad potential to study the biological characteristics of the cell of interest. A culture system allows to dissect the intrinsic processes controlling different cell functions and to investigate cell interactions. This knowledge will serve as a standard to study pathophysiological mechanisms as well as to evaluate the potential of the cells of interest to become a tool for regenerative medicine, for tissue repair using cell transplantation or cell mobilization in situ for cell replacement. Successes were achieved for epithelial cells of the skin [1] or the cornea [2], but innovative protocols have to be established for many organs with a complex structure.

The large diversity of the cell populations composing the central nervous system brings a new challenge to the generation of specific neuronal phenotypes. Several groups have attempted and continue to strive to generate fully differentiated neurons in a culture dish such as dopaminergic neurons, motoneurons, serotoninergic neurons and photoreceptors for instance. In this review, we will focus on the advances made in neuroscience that have favored the strategic development of new technologies to drive the generation of specific CNS neuron phenotypes and on photoreceptor production from stem cells.

One gold standard, to categorize a cell identity or to evaluate the efficacy of cell commitment from stem cells, is the transplantation challenge to determine whether the isolated cell phenotype can integrate its population in vivo and participate to a physiological function, the pioneer field using this approach being immunology [3]. Concerning the CNS, homotopic transplantation of neural cells attested that certain cell populations can integrate into their original brain region in a host recipient [4]. Concerning the retina, newly generated photoreceptors isolated from the retina during early development of the rods (between postnatal day (PN) 1 and PN7) and sorted by FACS can integrate into a retina after subretinal transplantation. These cells can migrate in the retina, fully mature, form synapses, mediate light stimuli and visual behaviors $[5,6]$. These results provide encouraging perspectives to repair the retina due to aging or genetic diseases inducing retinal degeneration such as age-related macular degeneration and Retinitis Pigmentosa respectively.

\section{Neural Cells and Neural Stem Cells}

Because neurons are postmitotic and because cell commitment occurs during the last or the ultimate cell division [7], researchers focused on more primitive cells able to renew in order to obtain enough available material for in vitro investigations. With the discovery of neural stem cells [8-11], several researchers attempted to generate a specific cell type by mimicking external signals occurring during their specialized commitment. Although in certain cases some characteristics of the desired phenotype were expressed, the full differentiation process

*Corresponding author: Yvan Arsenijevic, Hôpital Ophtalmique Jules-Gonin, avenue de France 15, 1004 Lausanne, Switzerland, Tel: +41 (0)21 62682 60; E-mail: yvan.arsenijevic@fa2.ch

Received May 21, 2015; Accepted July 08, 2015; Published July 10, 2015

Citation: Arsenijevic Y, Decembrini S (2015) An Advocacy for the Use of 3D Stem Cell Culture Systems for the Development of Regenerative Medicine: An Emphasis on Photoreceptor Generation. J Stem Cell Res Ther 5: 290. doi:10.4172/2157 7633.1000290

Copyright: (c) 2015 Arsenijevic $Y$, et al This is an open-access article distributed under the terms of the Creative Commons Attribution License, which permits unrestricted use, distribution, and reproduction in any medium, provided the original author and source are credited. 
did not occur. This lack of complete development is probably due the fact that each neural stem cell is already partially committed and necessitates the appropriate environmental stimuli to express markers of the late differentiation process [12]. These extrinsic factors remain largely unknown. Moreover, the neural stem cells are highly committed to the generation of glial cells in view of the low percentage of neurons generated from these cells $[10,13]$. Such gliogenic environment may also interfere with the neuronal development as well demonstrated by the co-culture of adult astrocytes with stem cells, the spinal cord glia inhibiting neurogenesis and the hippocampal astrocytes favoring neuron generation [14].

Concerning the retina, some groups focused on retinal progenitor cells during the perinatal period because the cells at this stage are committed to the formation of principally photoreceptors. These cells can extensively replicate $[15,16]$, are able to form glia and neurons in vitro and depending on the protocols can generate neurons expressing some retinal markers [15-17]. After transplantation of non-differentiated expanded retinal progenitors in different animal models, these cells tend to form glia and some rare neurons with retinal characteristics [16-19]. Cells expressing some photoreceptor markers were reported, but these transplanted cells never display a convincing complete maturation with the presence of well-formed outer segments and synapses with the target cells, the bipolar cells. This may be explained because the post-natal in vitro expanded progenitors, although expressing several transcription factors characteristic of the retinal progenitors, lack $\mathrm{Rx}$ and Chx10 expression (unpublished data). The small eye size of the mouse may also be responsible for the release of some gliogenic factors due to the injury procedure. Cell transfer into the sub retinal space of the pig retina, indeed, gives a better yield of cells expressing rhodopsin and transducing [20], but they don't integrate into the retina. Nonetheless, laser injury creates a permissive environment that stimulates passaged (P5) mouse retinal progenitors to migrate into the retina after sub retinal transplantation and differentiate into photoreceptors. These cells mature and form outer segments and express specific proteins such as Rhodopsin and Recoverin. The morphology is very similar to normal photoreceptors. Interestingly in previous studies, the authors challenged many other animal models of retinal degeneration but never obtained a photoreceptor differentiation process of this quality, resembling normal photoreceptors. Nonetheless, the cell phenotype which is able to integrate into the retina remains unclear. Is it a pure retinal progenitor or a cell already undergoing differentiation due to the experimental process? Further investigations are necessary in order to understand how a subgroup of retinal progenitors can respond to environmental cues to repopulate the photoreceptor layers.

Few studies attempted to differentiate in vitro expanded retinal progenitors/stem cells prior to the transplantation. The exposure in vitro of retinal stem cell-like cells to different factors known to participate to photoreceptor development commits the non-differentiated cells towards a retinal neuron fate [16,17] including the photoreceptor fate. However, these cells express only few specific retinal markers and don't robustly integrate into the retina after cell transplantation $[16,18,21]$. Because the eye and the retina of certain batrachians and fishes continue to grow due to the presence of adult retinal stem cells located at the periphery of the retina, different groups investigated a similar possibility in the mammal eye. The pigment epithelium, located between the periphery of the retina and the ciliary body of the mouse $[22,23]$ and human $[24]$ eye, contains a cell population able to proliferate in vitro under mitogen stimulations. After differentiation, the cells form glial cells and neurons with some retinal markers. Despite the fact that during bird and mouse development RPE cells are able to generate in vivo a retina under different experimental conditions [25,26], the adult pigmented cells identified at the mammalian retina periphery in the ciliary epithelium reveal a restricted capacity to produce fully differentiated retinal neurons in vitro [27] or in vivo after transplantation [24]. The pigmented cells of the mouse pars plana and plicata suffer in vitro from a pronounced chromosome instability appearing after few passages [28]. Such characteristic remains unknown in human cells which display in general a better stability in vitro. Supplementary signals or chromatin remodeling are probably necessary for the generation of bona fide retinal neurons from the adult pigmented cells. Indeed, the Chx10, Crx, Otx2 gene transfer into the adult ciliary epithelial cells improve cell differentiation into the photoreceptor fate [29].

\section{Embryonic Stem Cells to Generate Retinal Neurons}

The first impressive work showing the feasibility to commit ES cells into different retinal cell types used the co-incubation of mouse ES cells onto a layer of the PA6 stromal cell line [30]. Rapidly, clusters of cells grow as a tissue in 11 days and form a lens surrounded by pigment epithelial cells. Once the lens was cut for histology, neural cells were identified and found intermingled in the lens. Cells expressing 4 different retinal markers, including Rhodopsin, were revealed. Another approach considered the neural fate induction by using Noggin to inhibit the BMP pathways and then replating the cells in the presence of FGF-2 and EGF to form "neurospheres" [31]. These cells are committed to a neural fate and under the differentiation process, which consists in mitogen removal and plating onto a laminin substrate, they generate neurons and glia. RT-PCR analysis suggests that the neural progenitor in the neurospheres may have some characteristics of retinal progenitors and that differentiated cells may have the potential to generate retinal cells. Such ability was challenged by transplantation. No retinal neurons were integrated into the host retina, nonetheless cells expressing photoreceptors markers were identified in the subretinal graft [31].

These two studies show that environmental conditions can drive ES cells to express at least some retinal markers and that further stimuli are needed to generate fully differentiated cells and eliminate embryonic stem cells.

Because the embryonic stem (ES) cells are at a very naïve state, without exposure to stimuli inducing a specific fate, studies using sequential stimulations of ES cells with factors known to be key players during tissue morphogenesis and cell commitment rapidly brought new insights on the signals necessary to generate retinal neurons. The succession and the roles of different transcription factors during retinogenesis are now well documented and can serve as good markers to follow ES cell commitment and differentiation in vitro. However, the influence of the environment and extracellular matrix (ECM) proteins is much less understood. In order to bypass this lack of knowledge and to attempt to mimic the normal environment, the ES cells were cultured as serum-free floating culture of embryoid body-like aggregates (SFEB, [32]). The use of Dkk1 and Lefty to antagonize the activation of Wnt and Nodal pathways respectively greatly favors the induction of a rostral fate with the appearance of telencephalic cell phenotypes [33]. Indeed, Wnt and Nodal were observed at this cell stage to inhibit neural differentiation and Wnt to favor caudalization. The addition of $5 \%$ FCS and Activin markedly increases the percentage of colonies expressing $\mathrm{Rx}$ and Pax6 which are transcription factors specifically co-expressed in the early retinal lineage [32]. When the aggregates are transferred onto adherent substrates (poly-D-lysin with laminin and fibronectin) and the previous factors removed with the exception of Activin for 1 more day in culture, the cells progressively express photoreceptor markers such as Crx and then Rhodopsin. At the moment the percentage of 
cells acquiring this phenotype is low $(<0.5 \%$ of the total cells). Gene transfer mediated by lentivirus carrying the Crx transcription factor markedly enhances the production of Rhodopsin-positive cells. Interestingly, when these cells are cultivated in contact with embryonic retina explants, some cells migrate to the correct layer and develop outersegments [32].

This study shows the feasibility to generate photoreceptors from mouse ES cells that have the capacity to integrate into the retina after in vitro cell transfer. Further characterization is necessary to document to which extent these cells can undergo the photoreceptor differentiation process. The yield of retinal neurons at the end of the protocol is still very low, necessitating new protocol optimizations to generate enough material for deeper investigations. Nonetheless, this study strongly opened the way to use ES cells to generate retinal neurons and was the basis of several other studies. Moreover the use of the SFEB protocol is probably a key determinant to instruct the cells into the desired phenotype by giving a first $3 \mathrm{D}$ environment at the beginning of the culture system, allowing possibly the production of the correct extracellular matrix signals.

Using the SFEB protocol and cell sorting to isolate mouse retinal progenitors generated during the eye-field induction, Osakada et al. [34] tested different factors known to participate to the photoreceptor induction, survival and maturation. The addition of FGF-1, FGF2, Taurine, Shh and RA significantly increased the production of Rhodopsin-positive cells. This population represents $15 \%$ of FACS purified $\mathrm{Rx}$-positive retinal progenitors. The Rhodopsin-positive cells express many markers specific to the photoreceptor lineage. However, the yield in comparison to the number of original cells is quite low. Similar results were obtained with human ES cells but necessitated much more time for the differentiation process, 140 to 200 days to acquire protein expression of mature photoreceptors. Unfortunately, the transplantation of the mouse photoreceptors generated by this protocol into different mouse models of retinal degeneration did not allow integration into the retina, even in the wild-type animals [35] indicating that other signals are necessary to drive the retinal progenitors derived from ES cells to acquire a fully adult photoreceptor phenotype.

In a similar strategy to inactivate the inhibitors of forebrain induction, Lamba et al. [36] incubated human embryoid bodies with Dkk1 and Noggin for 3 days to block the Wnt and the Bmp pathways respectively. IGF-I was added to promote a retinal fate confirmed by the expression of 5 transcription factors essential for the early retinal progenitor identity. Then the cells were transferred for 1 to 3 weeks onto a Matrigel or Laminin substrate in the presence of FGF-2. Gene expressions of the differentiated cells are, for certain genes, very similar to this of a 91 day fetal human retina. Some genes are less expressed after the differentiation protocol, but nonetheless well present. The co-culture of the retinal progenitors derived from the human ES cells with retina explant of the Aipl1 ${ }^{-1-}$ which is affected by a strong retinal degenerative process, markedly increased the appearance of cells expressing photoreceptor markers such as Recoverin, Rhodopsin and Nrl.

When the human retinal cells derived from the above described differentiation protocol [36] were transplanted into newborn mice or into a mouse model of Leber Congenital Amaurosis due to a deficiency in the Crx gene, the cells migrated into the retina and differentiated into various retinal neurons including photoreceptors [37]. The donor cells were labeled with a lentiviral vectors 5 to 7 days before the cell transfer. The newly generated photoreceptors harbor well-formed outer segments expressing Rhodopsin. In several transplanted cells, the GFP expression was lost, but the human origin can be detected with an antibody against the human $\mathrm{Nrl}$ antigen. This study confirms the potential to use ES cells to generate retinal neurons. This study is also the first proof-of-concept that retinal cells derived from ES cells can incorporate the retina tissue after cell transplantation. However, because all the cells incubated in the differentiation medium were transplanted, it is not clear which cell type can integrate into the retina after transplantation and generate photoreceptors.

Concerning mouse ES and iPSCs, the different combinations of the factors Dkk1, Noggin, FGF-2, IGF-I, DAPT and FGF-1 during a stepwise protocol and during the course of ES-cell differentiation confirm the possibility to largely commit the pluripotent cells towards the retinal fate [38]. The transplantation of the differentiated cells also shows the capacity of the induced cells to integrate into the retina, but the morphology of the donor photoreceptors seems to be still immature after cell transfer, necessitating further stimuli to fully differentiate. Indeed, the transplanted cells lack long outer segments. Nonetheless, c-Fos activity was detected in the nucleus of cells composing the retinal inner nuclear layer (INL) suggesting that stimulations are received by photoreceptors under light exposure. Nonetheless, a potential trophic effect stimulating c-Fos in INL cells mediated by the graft was not investigated.

These series of experiments demonstrate that ES cells can be instructed to generate retinal cells expressing several specific characteristics and which are capable of morphologically integrating the retina after transplantation. The efficacy of ES cell commitment seems to be linked to the generation of embryoid bodies or aggregates named SFEB for serum-free, floating culture of embryoid body-like aggregates. Nonetheless, the retinal neuron yield has to be improved and tracked to be able to study the cell types at different development stages and to test their potential to repair an injured or degenerating retina.

\section{Towards a 3D Culture System to Generate Appropriate Neuronal Cells}

\section{The importance of cell interaction}

During this period, the generation of other neurons from the CNS mainly improved when techniques of neural tube induction, the rosette formation, was used to mimic the first stage of the neural tube organogenesis. ES cells were aggregated to form embryoid bodies in floating conditions, and cell attachment in the presence of laminin and insulin leads to the appearance of some neural progenitors. The addition of FGF-2 stimulates the proliferation of the neural progenitor in rosette-like configurations which nonetheless lack a fully arranged neuroepithelial structure [39]. The exposure of the cells to factors known to be involved in the differentiation of the dopaminergic neurons during development induces the appearance of the expected phenotype [40]. A similar strategy was used to form rosettes from hES which are then able to generate neurons, astrocytes, and oligodentrocytes [41]. After the transplantation of the neural precursors into the lateral ventricles of newborn mice, the cells migrated to different brain areas and morphologically integrated into the parenchymal tissue, such as the cortex, or the hippocampus for example [41].

In order to better master the cell specification of rosettes towards different CNS regional fate, the rosette formation step was improved by controlling its growth and maintenance by using several factors. Shh and FGF8 were found to promote efficiently the generation of rosettes as well as the expression of the forebrain marker BF1. By contrast 
the expansion and the maintenance of these rosettes necessitated the substitution of FGF-8 by Notch ligands such as Jagged and Delta4 [42]. The rosettes form lumens which are modeled with the presence of adhesion molecules such as the Zona Occludin- 1 and the N-Cadherin. Moreover, the BF1-positive population can be re-specified to more caudal CNS regions under the appropriate stimuli, to generate for instance Tyrosine hydroxylase-positive neurons of the midbrain or motoneurons of the spinal cord after differentiation. Moreover, these in vitro-generated neurons transplanted into the brain or the spinal cord of adult rats can survive and integrate morphologically into the CNS parenchyma and differentiate into TH neurons and motoneurons [42].

Similarly, another work used the above-mentioned protocol to induce rosette formation [41] with the following modifications. The neuralized region was dissected, isolated and stimulated to form floating neurospheres which were then plated onto laminin substrate with FGF-2 and EGF to allow their long-term propagation [43]. Standard characterization shows that these renewable cells have properties of neural stem cells. The cells maintain their phenotype with transcription factors specific of neural stem cells (or of rosettes when induced) and their ability to generate neurons and glia. The propagated cells also maintain a ventral hindbrain regional specificity all along their passaging. Interestingly, these cells can nonetheless respond to other factors to commit their fate to a ventral midbrain phenotype (such as the dopaminergic neuron) or to a ventral spinal cord phenotype (the motoneurons). The transplantation of long-term propagated neural stem cells derived from ES cells into the telencephalon of newborn mice allows the differentiation of certain neural progenitors into neurons. Electrophysiological recordings reveal that after 18 to 24 weeks of differentiation and maturation, neurons can receive input from the host brain.

The above experiments revealed the possibility of generating a specific neuron phenotype from ES cells by maintaining a close contact within a homogenous population of neural progenitors forming the rosette. Some of the progenitors or neurons produced can integrate into the brain parenchyma after transplantation attesting that these cells have correctly replicated some stages at least of the neural development. The prospect of controlling a whole tissue formation in vitro as an organoid structure is a supplementary step to better recapitulate, in a coordinate manner, the generation of the cells composing the tissue of interest. Different works aimed at inducing cortical neurons and other tissues contributed to important progresses in this field and are presented below.

\section{The formation of brain specific tissues: the cortex}

The self-aggregation of single dissociated cells in a non-adherent well and in absence of serum and growth factors (called SFEB for "serum-free, floating culture of embryoid body-like aggregates) induces the generation of a neural cells expressing Sox1. Because Wnt and Nodal favor the ES cell commitment to a non-neural fate, the next logical step was the addition of their respective antagonists, Dkk1 and Lefty, to improve neural induction and telencephalic fate as attested by the specific Bf1 transcription factor expression [33]. In this culture condition, cells form clusters. ES cell aggregation in a non-adherent substrate with a defined cell number provides the environment necessary for the cells to start self-organization into cortical tissue as confirmed by the expression of the Bf1 and Emx1 specific markers [44]. However, the cells rapidly form rosettes, disrupting the tissue homogeneity. The isolation of the Bf1-positive cells and their re-aggregation using different factors allows driving the cortical cells to adopt different regional identities. In order to maintain the cortical structure with a normal layering of the different neurons composing this brain region, the addition of Laminin and Entactin in the culture markedly enhances the stability of cortical tissue integrity [45]. Interestingly, the induction of human ES cells to the cortical tissue morphogenesis is more efficient with less disrupting regions [44].

Similar successes were obtained in the generation of cerebellar $[46,47]$ as well as hypothalamic tissues [48], the adenohypophysis organoid able to secrete pituitary hormones after stimulation [49] and the inner ear epithelium [50].

\section{The Development of a 3D Culture System to Recapitulate Tissue Organogenesis}

The 2D culture condition in monolayer creates an artificial environment which does not mirror the conditions encountered during the natural development where each organ develops as a $3 \mathrm{D}$ structure with the coordinate formation of various cell types under intrinsic and environmental cues. In order to mimic at best in vitro tissue development, different research groups have elaborated 3D culture systems to initiate the process of tissue formation by exposing these native buds to successive stimulations. Two marked advances are summarized below and for a deeper description of the molecular mechanisms as well as other examples, please refer to the review of Sasai's group [51]. Nonetheless below is briefly overviewed the pioneer work on the $3 \mathrm{D}$ culture system made on the gut as well as the success of 3D for kidney modeling.

\section{The gut}

The identification of the crypt-base columnar $(\mathrm{CBC})$ cells positive for the cell surface protein Lgr5 in the gut [52] allowed clearly defining this population as stem cells of the crypt gut responsible for the constant repopulation of the intestine epithelium. Nonetheless, this cell population is dispensable and even after its ablation the $4+$ cells can compensate for its loss [53]. The culture of one single Lgr5-positive cell, on Matrigel and in the presence of EGF as well as R-spondin-1 and Noggin, generates a gut organoid composed of crypts and microvilli [54]. The distribution of the different cell phenotypes in these microstructures corresponds to the morphology of the normal intestine. This example shows that a single cell in suspension in Matrigel can recapitulate the whole development of an epithelium composed of multiple cell types. The in vitro stimulation of LGR- 5 positive cells of the adult colonic crypt necessitates additional factors such as Wnt3a, hepatocyte growth factor (HGF) and BSA. Within this growth factor cocktail, EGF, Noggin and HGF are dispensable but sustain the organoid growth which forms a colonic crypt. The transplantation of these organoids was then tested in immunocompromised Rag $2^{-1-}$ mice. These animals were chemically injured to damage the colonic epithelium leading to diarrhea and body loss. The infusion of the organoids with Matrigel and PBS in the colon cavity allowed the donor cells to integrate into the host epithelium and to form characteristics cystic structures. The organoids treated animals recuperate their body weight more rapidly than the shame group. Colon repair from a single cell-derived culture was also demonstrated in this study [55]. Similar studies were performed with LGR5-positive stem cells form the different region of the small intestine. However, the intestinal stem cells could be isolated based on their low expression of LGR5 and by using Wnt antagonist. The Wnt activation signal then served to mature the organoids [56].

These series of experiments demonstrate the feasibility of restoring the function of various intestinal structures by using adequately differentiated cells derived from well characterized stem cells. 


\section{The kidney}

The kidney is a complex organ composed of different cell populations and structures. In a similar strategy as used for the gut, the identification of the progenitors at the origin of the metanephric nephron was a key element to produce in vitro kidney structures in organoids. Indeed, the characterization and the isolation of the Osrl early marker of the kidney development allowed generating a knock-in mouse expressing GFP to track this cell population during development [57]. In this Osr1-positive population, the co-expression of the integrin-a 8 in absence of Pdgfra defines the colony forming nephron progenitors. Embryoid bodies derived from ES-cells obtained from the Osr1-GFP mice were submitted to different stimulations occurring during the formation of the posterior mesoderm. The successive applications of Activin, Bmp4 and the Wnt agonist CHIR induce the appearance of the posterior nascent mesoderm markers. The combination of these factors with retinoic acid drives the cells to the intermediate mesoderm fate and the stimulation of the Wnt and Fgf9 pathways leads to the formation of cells expressing markers of the metanephric mesenchyme. Then the embryoid bodies were placed in contact with spinal cord or tissue to induce the organoid formation. This co-culture leads to the formation of tubules- and glomerulus-like structures with well- defined tissues similar to those present during the kidney development. The transplantation of the pre-conditioned EB with spinal cord tissue into the kidney capsule also leads to the formation of renal structures.

These two examples clearly show that the 3D culture system allows very primitive progenitors at the origin of an organ formation to recapitulate in vitro the main patterns of cell organogenesis to generate organoids closely resembling organ development.

\section{The 3D Culture System allows the Generation of bona fide Photoreceptors}

Concerning retinal cell generation, one of the most comprehensive reports which attempted to maintain cells in a $3 \mathrm{D}$ culture system throughout the procedure has been published by the group of Gamm [58]. In such protocol free-floating hES cells and hiPSC aggregates were driven toward neuroectodermal specification through the rosette formation on laminin-coated culture dishes and in a chemically-defined medium based on the N2 supplement. After 16 days of differentiation, adherent rosette-containing colonies mechanically removed to grow as neurospheres and expanded in a B27-based medium acquire the expression of transcription factors associated with eye field specification (Pax6, Rx, Six3, Six6, Lhx2, Tll) and with the anterior neural specification (Otx2) as well as markers of immature photoreceptors (Crx and Recoverin) from day 80 onward. Taking advantage of this multi-step protocol combining suspension and adherent cell cultures, Meyer et al. confirmed the possibility of employing hES cells able to produce an enriched population of photoreceptor cells and RPE at the earliest stage of retinal specification that closely mimics the key developmental stages of retinogenesis. Later on, other papers published by the same group validated these results $[59,60]$.

In 2011, the first striking protocol using a 3D culture system during the whole experiment has been developed by the Sasai's group to differentiate mouse ES into optic cups forming well-structured retina tissues [61] (Figure 1). This masterwork has been briefly followed by a second one exclusively focused on human ES [62]. These data demonstrated that several key features of retinogenesis can be recapitulated by $3 \mathrm{D}$ culture of mouse and human ES or iPSCs. To generate neuroepithelium with eye-cups, a defined number of pluripotent cells per well have to be used to make aggregates in 96-well plates. This step reveals that cell interactions and probably diffusible factors are crucial for the launch of the retinogenesis programme. The addition of factor-reduced Matrigel (FRM) during the first or the second day of differentiation in mouse and human pluripotent cells respectively, allows the self-organization of the retina neuroephitelium which rapidly evaginates to produce the optic vesicles that in turn invaginates to form the optic-cups. The major difference between the mouse and the human protocol is the use of the IWR1 Wnt inhibitor to counteract the caudalization effects due to the Knock out serum (KSR) high concentration that was needed to better induce the human retinal neuroepithelium formation. During the optic vesicle formation, the cells express the retinal progenitor transcription factors Rx, Pax6, and are negative for Sox 1 . Once generated, the optic cups were excised from the mother aggregate and left to maturate in suspension in a medium based on FBS, all-trans retinoic acid and L-taurine. This induced tissue then recapitulates the major features of the mammalian retinogenesis. The final tissue consists on a retina-like structure composed of several layers of photoreceptors, glial cells, the bipolar cells, making the connections between the photoreceptors and the ganglion cells, the interneurons involved in the modulations of the signal transmissions (amacrine and horizontal cells), and finally the ganglion cells. All the cells are arranged in a normal layering. Similar work with slightly modified protocol confirms the interest to generate retina cells from a $3 \mathrm{D}$ culture system [63]. The capacity of such retinas to mediate light stimuli was not yet assessed. It would be interesting to test this capacity since the photoreceptor maturation reached a growth limit with no formation of outer segments. Interestingly, the formation of outersegment was shown to be dependent on the action of the miR-182 and miR183 and overexpression of these miRNA in developing eye-cups derived from induced pluripotent cells (iPSCs) leads to the induction of outer segment buds able to mediate light-evoked responses [64].

The capacity of the $3 \mathrm{D}$ culture to recapitulate the retinogenesis events suggests that the retinal cells initiate a full program of differentiation although the final maturation of photoreceptors was not achieved in this system. In order to test that photoreceptors can generate outer segment and express correctly proteins of the phototransduction pathways as well as proteins composing the photoreceptor structure, different groups tested the capacity of in vitro-derived photoreceptors to integrate into a recipient retina after transplantation into adult retinas [65-67].

In the study of Gonzales-Cordero [67] ES-derived photoreceptor precursors were labeled with an AAV2/9-Rhodopsin-GFP vector 4 to 12 days prior cell transfer to track maturing photoreceptors. . Flowsorted rods were then transplanted into the subretinal space of adult mouse models of retinal degeneration $\left(\mathrm{Gnat}^{-/}, \operatorname{Prph} 2^{\mathrm{rd} 2 / \mathrm{rd} 2}\right.$, and the $\mathrm{Rho}^{-/}$). Based on gene expression (GFP), cells isolated from day 26 of culture presented an immature rod photoreceptor phenotype and integrated better than those isolated from late culture stages such as day 34. The biological capacity of the former-mentioned cells resembles that of freshly dissociated Nrl-GFP-positive photoreceptor precursors collected from postnatal day 4 retinas. This age corresponds to the best photoreceptor differentiation stage for transplantation $[5,6]$.

In close agreement with Gonzalez-Cordero's work, the transplantation of early-born photoreceptors generated from an ES cell line expressing GFP under the control of the Crx specific photoreceptor promoter gives similar results $[66,68]$. This study generated a fine-tuned $3 \mathrm{D}$ culture system to track developing photoreceptors in the dish. The advantage of the Crx-GFP cell line is to track the cells to study their biology, their commitment to the photoreceptor fate, and to avoid vector 


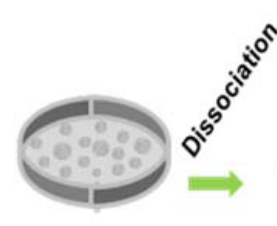

(A)

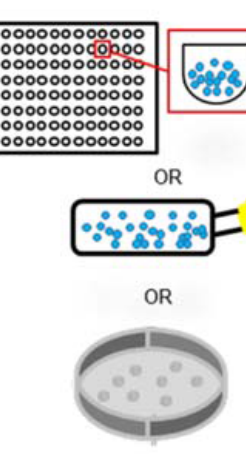

(B)

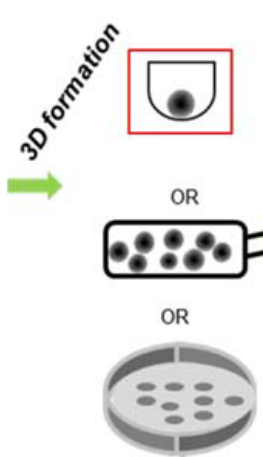

(C)

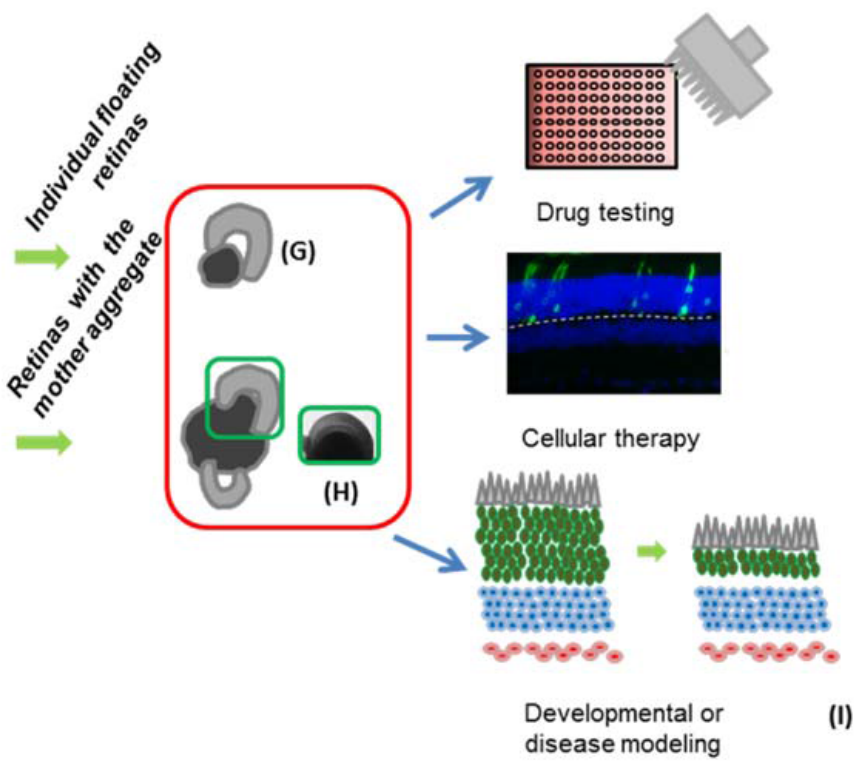

Figure 1: From pluripotent stem cells to $3 \mathrm{D}$ retinas. A) Human pluripotent stem cells are (B) dissociated and seeded in specific medium conditions in order to form either aggregates in 96-low binding plates, embryoid body-like structures in flasks, or pluripotent cell colonies on matrigel or other extracellular matrix (ECM) proteins. (C) Depending on the technique used, seeded cells will generate embryoid body-like structures or floating/adherent aggregates. (D) The induction of a neuroectodermal ring surrounding the aggregate drives $(E)$ the formation of optic vesicles (patterning) which in turn invaginate to form ( $F$ ) optic cups (morphogenesis). Optic cups develop inside the aggregate or are cut away. In other 2D protocols they are directly detached from the substrate. These two different modi operandi give rise to individual floating retinas $(\mathrm{G})$ or to aggregate-bearing retinas $(\mathrm{H})$. (G-I) These retina-like tissues can be used for modeling retinal developmental and degenerative diseases, as well as for testing cellular therapy and pharmacologic agents and gene transfer. In (I), green cells are photoreceptors, blue and pink ones are interneurons and ganglion cells respectively.

use to label the cells, such transduction rendering fragile the cells during some days. In both studies the number of integrated cells is similar and the photoreceptors undergo a full maturation with the formation of long outer segment containing proteins of the photo transduction pathway as well as specific proteins necessary for the outer segments structure. The inverse translocation movement of Arrestin and Transducin (GNAT1) under light exposure reveals that the transplanted cells have a certain capacity to respond to light stimuli [66]. Interestingly, in both studies, the transplanted photoreceptors form synapses with the adjacent bipolar cells. Moreover, the transplanted photoreceptors can modulate their intracellular $\mathrm{Ca}^{++}$flux in function of mGluR8 activation and inhibition, as the host photoreceptors [67]. These studies show that the transplanted cells respond normally to their local environment. Importantly, due to the low numbers of integrated cells, no visual function restoration assessments were attempted in these studies.
Using an Rx-KI-GFP mES cell line [34] to label cells committed to the eye-field fate, the 3D culture system was improved by adding retinoic acid receptor antagonist (RRA) AGN193109 to inhibit cell fate caudalization and by increasing the knockout serum replacement (KSR) concentration (from $1.5 \%$ to $5 \%$ ) to produce larger quantities of neural retinal tissue for transplantation [65]. Using this protocol, RxGFP mES formed aggregates strongly expressing Rx-GFP on day 8 in optic vesicle-like structures which were transplanted as retinal sheets after different days of culture. With this experiment the authors showed that transplanted 3D differentiated mES- or miPSC-derived retinal sheets could develop to form ONL structures consisting in mature photoreceptors that were indistinguishable in their morphological development from those of the endogenous wild-type ONL. These transplanted photoreceptors could also form complete inner- and outer segments. After transplantation, the grafts of day 17 or younger were 
the most efficient in forming such structured ONLs and making direct contact with host INL. The advantage of such protocol in comparison to single cell transplantation is to deliver a tissue layer that may result in a better visual acuity if the graft is well integrated into the host retina circuitry.

These three papers showed that despite the use of different protocols, diverse recipient mice and distinct pluripotent lines, ES cell lines allowed the generation of retinal tissues capable of precisely recapitulating the in vivo mouse development. Moreover, the pluripotent cells coupled with the $3 \mathrm{D}$ culture provide a renewable source to generate bona fide photoreceptors that can migrate and integrate into the ONL of adult recipient retinas after transplantation, displaying the morphology of mature rod photoreceptors, such as the presence of complete outersegment growth and forming synaptic contacts with the endogenous bipolar cells.

Concerning the generation of human photoreceptors, several protocols were successful to produce optic cups containing photoreceptors from pluripotent cells [62,69]. Interestingly, photoreceptors can mature and form tiny outersegments. Although these structures are just forming, patch clamp recordings revealed that some cells are able to respond to a single light flash.

These series of experiments show that newly formed photoreceptors can normally differentiate, undergo maturation and respond to light stimuli highlighting the potential of these cells to be used for retina repair and to study their development.

Another noteworthy protocol has been reported by Knoblich's laboratory. Lancaster et al. described for the first time the creation of a three-dimensional culture modelling of the developing human brain [70]. The neuroectoderm was generated from EBs following modifications of the protocol published by Xia and Zhang [71]. Then tissues embedded in droplets of Matrigel were transferred to a spinning bioreactor to enhance nutrient absorption. This method led to rapid development of brain tissues, namely cerebral organoids, which reached maximal size by 2 months. Morphological and histological analysis revealed regions reminiscent of cerebral cortex, choroid plexus, retina and meninges as well as the patterning of the forebrain, midbrain and hindbrain regions. Impressively, despite the absence of a clear estimation of the number of retinas generated, of the degree of retina maturation reached and of the percentage of retinas ensheated by RPE, the pictures provided by Lancaster et al. showed astonishing layered retinas enfolded by RPE. These results along with the innovative technique which takes advantage of the bioreactor to improve nutrient absorption as well as $\mathrm{O} 2$ circulation in vitro and the generation of the eye and brain structures render this protocol worth mentioning for further investigations.

\section{Perspectives}

\section{Dissecting cell biology in organoid formation}

During several decades biologists attempted to isolate and cultivate different cell types in a controlled environment to study their biology and provide knowledge for cell-based therapy. Several cell lines were generated or protocols developed to isolate primary cells in order to investigate them during a short time period providing data of high value.

However, the restricted amount of cells available for one experiment (for instance the inner hair cells), the difficulty to control their survival (i.e. the photoreceptors), and the diversity of the protocols used prevent a rapid progress in the understanding of biological processes. Without disclaiming the important value of the $2 \mathrm{D}$ cell culture experiments at the basis of important discoveries, the 3D culture system brings a new innovative way to study cell biology and to instruct cells to recapitulate the development of a specific tissue or one single cell phenotype. This methodology will allow to better dissect the regulation of certain genes and pathways as well as identifying new cell phenotypes participating to tissue organogenesis. In this context, the elucidation of the role of extracellular matrix (ECM) proteins during morphogenesis remains an important challenge. The relative "easiness" to generate now organoids recapitulating tissue development should not hide the importance of the cell interactions allowing such organization. The molecular mechanisms of ECM proteins controlling cell organogenesis and fate will provide new tools to better form these organoids and use them for cell/tissue transplantation. Moreover, many of the protocols used to generate cell tissues are based on stimulation with Matrigel which contains several unknown factors derived from the tumor cell line producing this gel. In consequence, the identification of factors initiating organogenesis and allowing the development of organoids is of prime importance to further control the generation of these tissues.

\section{The importance of studying organ development}

The success of organoid formation relies on the identification of both the cell type to stimulate and the factors involved in this stimulation. From the different studies described in this review, cells isolated during development or after pluripotent cell commitment may have the potential to launch a morphogenic program if their molecular and structural phenotype is well defined and appropriately introduced in the in vitro system to recapitulate the developmental features. Because pluripotent cells can be instructed to build a cell tissue undergoing similar processes occurring during development, the deep analysis of the organ development is a major key element to using organoid generation in a perspective of regenerative medicine. In that context, the method to derive iPSCs from somatic tissue is also critical to derive cells having the closest similarity with the normal cells [72].

\section{Cell and tissue transplantation}

The identification of the different cell actors involved in organ morphogenesis and the understanding of the molecular events controlling their function are critical in order to isolate the cells able to integrate into a tissue after transplantation or to create an organ in the future. Several steps have to be reached before cells can be characterized for a standardized protocol of cell transplantation. First the cell production has to be compatible with a clinical application by avoiding xenogeneic material and compounds that cannot be reliably produced. The possibility to generate large numbers of cells from pluripotent cells will help to identify factors compatible with good manufacturing processes (GMP). Second, depending on the protocol used a heterogeneous cell population may be generated around the cell of interest for the transplantation. Protocols allowing reliable cell isolation have to be developed by isolating a pure cell population of interest and by eliminating any cells having undesired effects or tumorigenic potential. In that context, the identification of an extracellular marker for cell isolation is of high interest. Third, the isolated cells have to be reliably transferred into the diseased tissue to be repaired. The characterization of the disease course altering the host tissue has to be carefully investigated to evaluate whether and when the organ environment is suitable to accept a new tissue. For instance, transplantation of photoreceptor precursors into the normal retina or in the Gnat $1^{-1-}$ mouse model of retinal degeneration allows a good 
integration of the transplanted cells. However, several other models of retinal dystrophies, such as $\mathrm{Rho}^{-/}$[73], prevent efficient donor cell integration due to an important gliosis in the retina and the maintenance of the retina integrity structure. In Gnat $1^{-1}$ mice the glia is weakly activated and the outer-limiting membrane formed by the Muller cell feet adhesion molecules is altered allowing a good penetration of the transplanted cells injected subretinally. The potential therapeutic window for cell transplantation is difficult to evaluate in vivo, because the gliosis progresses variably during the degenerative process. These examples reveal the necessity to characterize the environment of the host tissue to optimize cell integration after transplantation (for a deep description of the transplantation of photoreceptors, please refer to Jayakodi et al. [74]). Fourth, the immunogenecity of the cells to transplant has to be investigated to attempt to minimize inflammation and the immunoreaction of the host.

\section{Modeling diseases with pluripotent cells}

Besides the development of organ repair, the iPSC technology can also potentially contribute to health research by offering the possibility to model human diseases in vitro and by providing the biological material for high throughput drug screening. iPSCs were derived from patients affected by different diseases [75]. In several cases, new pathological mechanisms were identified increasing our knowledge of the disease evolution which may serve to identify new therapeutic targets. The gene editing technology rend now more rapid the generation of cell lines with specific mutations using the CRISPR technology to model diseases rather than deriving new cell lines from patients. This technology provides advantages if the study focuses on revealing the function of one single gene. However, iPSC derivation from patients remains an interesting tool to study the pathophysiology of the diseased cells. Indeed, the mutated gene found to be associated with the disease may have also been co-segregated with some modifiers, which also play important roles during the course of the disease. Nonetheless, the combination of iPSCs and CRISPR techniques will dramatically increase our knowledge of disease mechanisms in a close future. Once disease models are validated, they can also serve for high throughput screening to identify drugs delaying the disease progress or to detect pathways controlling certain biological cascades. However, iPSC-dependent high-throughput drug screening is now still in early stage of development. Using organoids, several parameters need to be optimized and explored in more detail as the potential influence of the donor cell type, the detection of the cell or pathway of interest, the evaluation of gene editing off targets, the standardization of organoid growth, and the detection of possible aberrations.

\section{The necessity of iPS cell banking}

To allow scientist to make rapid progress in the different fields exposed here, cell banking and sharing is of high priority to prevent time waste to re-derive cell lines bearing the same characteristics as those already performed by other groups and to work with material that can be comparable from lab to lab. In this context, the initiatives of non-profit organizations such as the National Institute of Health (NIH) with the Center for Regenerative Medicine to derive iPSCs and bank them at the Coriell institute as well as WiCell, the iPS Core Facility of the Harvard Stem Cell Institute, and the European Bank for Induced Pluripotent Stem Cells (to name only some of them, see website references below) to render available several iPSC lines derived from healthy and patients will surely contribute massively to the iPSC research development [76]. Moreover, the access to a large number of iPSC lines will help to develop a better personalized medicine approach by being able to identify which genotype is responsive to a certain medication, and which ones are not [75]. This knowledge will also encourage pharmaceutical companies in the future to optimize clinical trials by better targeting the patient population of interest. This approach will contribute to avoiding side effects in patient population subtypes (non-responsive to the drugs), and allows the entry of drugs in the market that are beneficial for a certain population and not for others and, in consequence, should reduce health costs. The proof-ofprinciple that transplantation of cells derived from iPSCs is feasible and efficient will first be tested in an autologous condition as it was recently performed for the transplantation of RPE cells in a patient affected by age-related macular degeneration (http://www.riken-ibri.jp/ AMD/english/). However, the cost of such approach will be too high if generalized. Once the safety issues and efficacy are attested, a halogenic transplantation approach appears more realistic from an economic point of view. The characterization and the banking of the different iPSC haplotypes of healthy donors that are necessary for clinical applications with a perspective of cell transplantation will be of high value to propose compatible cells with the broadest immunological diversity of patients.

\section{Acknowledgments}

The authors thank Corinne Kostic and Dana Wanner for constructive comments when writing the review. Our work is supported by the Provisu Foundation, the Bertarelli Foundation, and the SwissTransMed subside of the CRUS

\section{References}

1. Sun BK, Siprashvili Z, Khavari PA (2014) Advances in skin grafting and treatment of cutaneous wounds. Science 346: 941-945. [PubMed]

2. Pellegrini G, De Luca M, Arsenijevic $Y$ (2007) Towards therapeutic application of ocular stem cells. Semin Cell Dev Biol 18: 805-818. [PubMed]

3. Spangrude GJ, Heimfeld S, Weissman IL (1988) Purification and characterization of mouse hematopoietic stem cells. Science 241: 58-62. [PubMed]

4. Campbell K, Olsson M, Bjorklund A (1995) Regional incorporation and sitespecific differentiation of striatal precursors transplanted to the embryonic forebrain ventricle. Neuron 15: 1259-1273. [PubMed]

5. MacLaren RE, Pearson RA, MacNeil A, Douglas RH, Salt TE, et al. (2006) Retinal repair by transplantation of photoreceptor precursors. Nature 444: 203207. [PubMed]

6. Pearson RA, Barber AC, Rizzi M, Hippert C, Xue T, et al. (2012) Restoration of vision after transplantation of photoreceptors. Nature 485: 99-103. [PubMed]

7. Cepko C (2014) Intrinsically different retinal progenitor cells produce specific types of progeny. Nat Rev Neurosci 15: 615-627. [PubMed]

8. Cattaneo E, McKay R (1990) Proliferation and differentiation of neuronal stem cells regulated by nerve growth factor. Nature 347: 762-765. [PubMed]

9. Palmer TD, Takahashi J, Gage FH (1997) The adult rat hippocampus contains primordial neural stem cells. MolCell Neurosci 8: 389-404. [PubMed]

10. Reynolds BA, Tetzlaff W, Weiss S (1992) A multipotent EGF-responsive striatal embryonic progenitor cell produces neurons and astrocytes. J Neurosci 12: 4565-4574. [PubMed]

11. Reynolds BA, Weiss S (1992) Generation of neurons and astrocytes from isolated cells of the adult mammalian central nervous system. Science 255 : 1707-1710. [PubMed]

12. Hitoshi S, Tropepe V, Ekker M, van der KD (2002) Neural stem cell lineages are regionally specified, but not committed, within distinct compartments of the developing brain. Development 129: 233-244. [PubMed]

13. Arsenijevic $Y$, Weiss $S$ (1998) Insulin-like growth factor-I is a differentiation factor for postmitotic CNS stem cell-derived neuronal precursors: distinct actions from those of brain-derived neurotrophic factor. J Neurosci 18: 21182128. [PubMed]

14. Song $H$, Stevens $C F$, Gage $F H$ (2002) Astroglia induce neurogenesis from adult neural stem cells. Nature 417: 39-44. [PubMed]

15. Angenieux B, Shorderet DF, Arsenijevic $Y$ (2006) Epidermal Growth Factor Is $A$ Neuronal Differentiation Factor For Retinal Stem Cells In Vitro. Stem Cells 24: 696-706. [PubMed] 
Citation: Arsenijevic Y, Decembrini S (2015) An Advocacy for the Use of 3D Stem Cell Culture Systems for the Development of Regenerative Medicine: An Emphasis on Photoreceptor Generation. J Stem Cell Res Ther 5: 290. doi:10.4172/2157-7633.1000290

16. Klassen HJ, Ng TF, Kurimoto Y, Kirov I, Shatos M, et al. (2004) Multipotent retinal progenitors express developmental markers, differentiate into retinal neurons, and preserve light-mediated behavior. Invest Ophthalmol Vis Sci 45: 4167-4173. [PubMed]

17. Merhi-Soussi F, Angenieux B, Canola K, Kostic C, Tekaya M, et al. (2006) High Yield of Cells Committed to the Photoreceptor Fate from Expanded Mouse Retinal Stem Cells. Stem Cells 24: 2060-2070. [PubMed]

18. Canola K, Angenieux B, Tekaya M, Quiambao A, Naash MI, et al. (2007) Retinal stem cells transplanted into models of late stages of retinitis pigmentosa preferentially adopt a glial or a retinal ganglion cell fate. Invest Ophthalmol Vis Sci 48: 446-454. [PubMed]

19. Canola K, Arsenijevic $Y$ (2007) Generation of cells committed towards the photoreceptor fate for retinal transplantation. Neuroreport 18: 851-855. [PubMed]

20. Klassen H, Kiilgaard JF, Zahir T, Ziaeian B, Kirov I, et al. (2007) Progenitor cells from the porcine neural retina express photoreceptor markers after transplantation to the subretinal space of allorecipients. Stem Cells 25: 1222 1230. [PubMed]

21. Chacko DM, Rogers JA, Turner JE, Ahmad I (2000) Survival and differentiation of cultured retinal progenitors transplanted in the subretinal space of the rat. Biochem Biophys Res Commun 268: 842-846. [PubMed]

22. Ahmad I, Tang L, Pham H (2000) Identification of neural progenitors in the adult mammalian eye. Biochem Biophys Res Commun 270: 517-521. [PubMed]

23. Tropepe V, Coles BL, Chiasson BJ, Horsford DJ, Elia AJ, et al. (2000) Retinal stem cells in the adult mammalian eye. Science 287: 2032-2036. [PubMed]

24. Coles BL, Angenieux B, Inoue T, Rio-Tsonis K, Spence JR, et al. (2004) Facile isolation and the characterization of human retinal stem cells. Proc Natl Acad Sci USA 101:15772-15777. [PubMed]

25. Spence JR, Madhavan M, Ewing JD, Jones DK, Lehman BM, et al. (2004) The hedgehog pathway is a modulator of retina regeneration. Development 131: 4607-4621. [PubMed]

26. Zhao S, Hung FC, Colvin JS, White A, Dai W, et al. (2001) Patterning the optic neuroepithelium by FGF signaling and Ras activation. Development 128: 5051 5060. [PubMed]

27. Cicero SA, Johnson D, Reyntjens S, Frase S, Connell S, et al. (2009) Cells previously identified as retinal stem cells are pigmented ciliary epithelial cells. Proc Natl Acad Sci USA 106: 6685-6690. [PubMed]

28. Djojosubroto M, Bollotte F, Wirapati P, Radtke F, Stamenkovic I, et al. (2009) Chromosomal number aberrations and transformation in adult mouse retinal stem cells in vitro. Invest Ophthalmol Vis Sci 50: 5975-5987. [PubMed]

29. Inoue T, Coles BL, Dorval K, Bremner R, Bessho Y, et al. (2010) Maximizing functional photoreceptor differentiation from adult human retinal stem cells. Stem Cells 28: 489-500. [PubMed]

30. Hirano M, Yamamoto A, Yoshimura N, Tokunaga T, Motohashi T, et al. (2003) Generation of structures formed by lens and retinal cells differentiating from embryonic stem cells. Dev Dyn 228: 664-671. [PubMed]

31. Banin E, Obolensky A, Idelson M, Hemo I, Reinhardtz E, et al. (2006) Retinal incorporation and differentiation of neural precursors derived from human embryonic stem cells. Stem Cells 24: 246-257. [PubMed]

32. Ikeda H, Osakada F, Watanabe K, Mizuseki K, Haraguchi T, et al. (2005) Generation of Rx+/Pax6+ neural retinal precursors from embryonic stem cells. Proc Natl Acad Sci USA 102: 11331-11336. [PubMed]

33. Watanabe K, Kamiya D, Nishiyama A, Katayama T, Nozaki S, et al. (2005) Directed differentiation of telencephalic precursors from embryonic stem cells. Nat Neurosci 8: 288-296. [PubMed]

34. Osakada F, Ikeda H, Mandai M, Wataya T, Watanabe K, et al. (2008) Toward the generation of rod and cone photoreceptors from mouse, monkey and human embryonic stem cells. Nat Biotechnol 26: 215-224. [PubMed]

35. West EL, Gonzalez-Cordero A, Hippert C, Osakada F, Martinez-Barbera JP, et al. (2012) Defining the integration capacity of embryonic stem cell-derived photoreceptor precursors. Stem Cells 30: 1424-1435. [PubMed]

36. Lamba DA, Karl MO, Ware CB, Reh TA (2006) Efficient generation of retinal progenitor cells from human embryonic stem cells. Proc Natl Acad Sci USA 103: 12769-12774. [PubMed]
37. Lamba DA, Gust J, Reh TA (2009) Transplantation of human embryonic stem cell-derived photoreceptors restores some visual function in Crx-deficient mice. Cell Stem Cell 4: 73-79. [PubMed]

38. Tucker BA, Park IH, Qi SD, Klassen HJ, Jiang C, et al. (2011) Transplantation of adult mouse iPS cell-derived photoreceptor precursors restores retinal structure and function in degenerative mice. Plos one 6: e18992. [PubMed]

39. Okabe S, Forsberg-Nilsson K, Spiro AC, Segal M, McKay RD (1996) Development of neuronal precursor cells and functional postmitotic neurons from embryonic stem cells in vitro. Mechanisms of development 59: 89-102. [PubMed]

40. Lee SH, Lumelsky N, Studer L, Auerbach JM, McKay RD (2000) Efficient generation of midbrain and hindbrain neurons from mouse embryonic stem cells. Nat Biotechnol 18: 675-679. [PubMed]

41. Zhang SC, Wernig M, Duncan ID, Brustle O, Thomson JA (2001) In vitro differentiation of transplantable neural precursors from human embryonic stem cells. Nat Biotechnol 19: 1129-1133. [PubMed]

42. Elkabetz Y, Panagiotakos G, Al Shamy G, Socci ND, Tabar V, et al. (2008) Human ES cell-derived neural rosettes reveal a functionally distinct early neural stem cell stage. Genes Dev 22: 152-165. [PubMed]

43. Koch P, Opitz T, Steinbeck JA, Ladewig J, Brustle O (2009) A rosette-type, self-renewing human ES cell-derived neural stem cell with potential for in vitro instruction and synaptic integration. Proc Natl Acad Sci USA 106: 3225-3230. [PubMed]

44. Eiraku M, Watanabe K, Matsuo-Takasaki M, Kawada M, Yonemura S, et al. (2008) Self-organized formation of polarized cortical tissues from ESCs and its active manipulation by extrinsic signals. Cell Stem Cell 3: 519-532. [PubMed]

45. Nasu M, Takata N, Danjo T, Sakaguchi H, Kadoshima T, et al. (2012) Robus formation and maintenance of continuous stratified cortical neuroepithelium by laminin-containing matrix in mouse ES cell culture. PloS one 7: e53024. [PubMed]

46. Muguruma K, Nishiyama A, Kawakami H, Hashimoto K, Sasai Y (2015) Selforganization of polarized cerebellar tissue in $3 \mathrm{D}$ culture of human pluripoten stem cells. Cell reports 10: 537-550. [PubMed]

47. Muguruma K, Nishiyama A, Ono Y, Miyawaki H, Mizuhara E, et al. (2010) Ontogeny-recapitulating generation and tissue integration of ES cell-derived Purkinje cells. Nat Neurosci 13: 1171-1180. [PubMed]

48. Wataya T, Ando S, Muguruma K, Ikeda H, Watanabe K, et al. (2008) Minimization of exogenous signals in ES cell culture induces rostral hypothalamic differentiation. Proc Natl Acad Sci USA 105: 11796-11801. [PubMed]

49. Suga H, Kadoshima T, Minaguchi M, Ohgushi M, Soen M, et al. (2011) Selfformation of functional adenohypophysis in three-dimensional culture. Nature 480: 57-62. [PubMed]

50. Koehler KR, Mikosz AM, Molosh Al, Patel D, Hashino E (2013) Generation of inner ear sensory epithelia from pluripotent stem cells in 3D culture. Nature 500: 217-221. [PubMed]

51. Sasai $Y$, Eiraku M, Suga $H$ (2012) In vitro organogenesis in three dimensions: self-organising stem cells. Development 139: 4111-4121. [PubMed]

52. Barker N, van Es JH, Kuipers J, Kujala P, van den Born M, et al. (2007) Identification of stem cells in small intestine and colon by marker gene Lgr5. Nature 449: 1003-1007. [PubMed]

53. Tian H, Biehs B, Warming S, Leong KG, Rangell L, et al. (2011) A reserve stem cell population in small intestine renders Lgr5-positive cells dispensable. Nature 478: 255-259. [PubMed]

54. Sato T, Vries RG, Snippert HJ, van de Wetering M, Barker N, et al. (2009) Single Lgr5 stem cells build crypt-villus structures in vitro without a mesenchyma niche. Nature 459: 262-265. [PubMed]

55. Yui S, Nakamura T, Sato T, Nemoto Y, Mizutani T, et al. (2012) Functional engraftment of colon epithelium expanded in vitro from a single adult Lgr5(+) stem cell. Nature medicine 18: 618-623. [PubMed]

56. Fordham RP, Yui S, Hannan NR, Soendergaard C, Madgwick A, et al. (2013) Transplantation of expanded fetal intestinal progenitors contributes to colon regeneration after injury. Cell Stem Cell 13: 734-744. [PubMed]

57. Taguchi A, Kaku Y, Ohmori T, Sharmin S, Ogawa M, et al. (2014) Redefining the in vivo origin of metanephric nephron progenitors enables generation of complex kidney structures from pluripotent stem cells. Cell Stem Cell 14: 5367. [PubMed] 
Citation: Arsenijevic Y, Decembrini S (2015) An Advocacy for the Use of 3D Stem Cell Culture Systems for the Development of Regenerative Medicine: An Emphasis on Photoreceptor Generation. J Stem Cell Res Ther 5: 290. doi:10.4172/2157-7633.1000290

Page 10 of 10

58. Meyer JS, Shearer RL, Capowski EE, Wright LS, Wallace KA, et al. (2009) Modeling early retinal development with human embryonic and induced pluripotent stem cells. Proc Natl Acad Sci USA 106: 16698-16703. [PubMed]

59. Meyer JS, Howden SE, Wallace KA, Verhoeven AD, Wright LS, et al. (2011) Optic vesicle-like structures derived from human pluripotent stem cells facilitate a customized approach to retinal disease treatment. Stem Cells 29: 1206-1218. [PubMed]

60. Phillips MJ, Perez ET, Martin JM, Reshel ST, Wallace KA, et al. (2014) Modeling human retinal development with patient-specific induced pluripotent stem cells reveals multiple roles for visual system homeobox 2 . Stem Cells 32: 14801492. [PubMed]

61. Eiraku M, Takata N, Ishibashi H, Kawada M, Sakakura E, et al. (2011) Selforganizing optic-cup morphogenesis in three-dimensional culture. Nature 472 51-56. [PubMed]

62. Nakano T, Ando S, Takata N, Kawada M, Muguruma K, et al. (2012) Selfformation of optic cups and storable stratified neural retina from human ESCs. Cell Stem Cell 10: 771-785. [PubMed]

63. Zhu Y, Carido M, Meinhardt A, Kurth T, Karl MO, et al. (2013) Three-dimensional neuroepithelial culture from human embryonic stem cells and its use for quantitative conversion to retinal pigment epithelium. PloS one 8: e54552. [PubMed]

64. Busskamp V, Krol J, Nelidova D, Daum J, Szikra T, et al. (2014) miRNAs 182 and 183 are necessary to maintain adult cone photoreceptor outer segments and visual function. Neuron 83: 586-600. [PubMed]

65. Assawachananont J, Mandai M, Okamoto S, Yamada C, Eiraku M, et al. (2014) Transplantation of embryonic and induced pluripotent stem cell-derived 3D retinal sheets into retinal degenerative mice. Stem cell reports 2: 662-674. [PubMed]

66. Decembrini S, Koch U, Radtke F, Moulin A, Arsenijevic Y (2014) Derivation of traceable and transplantable photoreceptors from mouse embryonic stem cells Stem cell reports 2: 853-865. [PubMed]

67. Gonzalez-Cordero A, West EL, Pearson RA, Duran Y, Carvalho LS, et al. (2013) Photoreceptor precursors derived from three-dimensional embryonic stem cell cultures integrate and mature within adult degenerate retina. Nat Biotechnol 31: 741-747. [PubMed]

68. Samson M, Emerson MM, Cepko CL (2009) Robust marking of photoreceptor cells and pinealocytes with several reporters under control of the Crx gene. Dev Dyn 238: 3218-3225. [PubMed]

69. Zhong X, Gutierrez C, Xue T, Hampton C, Vergara MN, et al. (2014) Generation of three-dimensional retinal tissue with functional photoreceptors from human iPSCs. Nat Commun 5: 4047. [PubMed]

70. Lancaster MA, Renner M, Martin CA, Wenzel D, Bicknell LS, et al. (2013) Cerebral organoids model human brain development and microcephaly. Nature 501: 373-379. [PubMed]

71. Xia X, Zhang SC (2009) Differentiation of neuroepithelia from human embryonic stem cells. Methods Mol Biol 549: 51-58. [PubMed]

72. Theunissen TW, Jaenisch R (2014) Molecular control of induced pluripotency. Cell Stem Cell 14: 720-734. [PubMed]

73. Barber AC, Hippert C, Duran Y, West EL, Bainbridge JW, et al. (2013) Repair of the degenerate retina by photoreceptor transplantation. Proc Natl Acad Sci USA 110: 354-359. [PubMed]

74. Jayakody SA, Gonzalez-Cordero A, Ali RR, Pearson RA (2015) Cellular strategies for retinal repair by photoreceptor replacement. Prog Retin Eye Res 46: 31-66. [PubMed]

75. Inoue H, Nagata N, Kurokawa H, Yamanaka S (2014) iPS cells: a game changer for future medicine. EMBO J 33: 409-417. [PubMed]

76. McKernan R, Watt FM (2013) What is the point of large-scale collections of human induced pluripotent stem cells? Nat Biotechnol 31: 875-877. [PubMed] 